\title{
Current Measurement System for Solder Joint Quality Analysis in Photovoltaic Modules
}

\author{
M. Lenzhofer ${ }^{1}$, L. Neumaier ${ }^{1}$, P. Malago ${ }^{1}$, J. Kosel and M. Ortner ${ }^{1}$ \\ 1 Silicon Austria Labs SAL GmbH, Europastraße 12, 9524 Villach, Austria, \\ E-mail: martin.lenzhofer@silicon-austria.com
}

\begin{abstract}
Summary:
This paper deals with a novel approach of using a multi frequency eddy current measurement system for analyzing the quality of solder joints in a photovoltaic (PV) module. Due to environmental conditions, thermal cycles and variable load situations the solder joints of the cell connectors inside a photovoltaic module suffer and properly lead to degradation or even worse to completely fail, which in turn can be accountable for burn marks, hotspots, arcing discharges and of course, substantial power losses. With the presented approach, the conductivity of each solder joint can be individually analyzed, enabling the possibility of early failure detection in-line or even in the field. The measurement results show that the evaluated states correlate to conductivity decrease of solder joints, which can be confirmed by electroluminescence (EL) images of a solar cell.
\end{abstract}

Keywords: Eddy current measurement system, photovoltaic module, solder joint inspection, conductivity measurement

\section{Introduction and Principle}

The photovoltaic (PV) technology plays an important role in the sector of renewable energy sources. Many companies and research institutes try to raise the efficiency of PV modules, to increase the power output and additionally reduce their manufacturing costs with novel technologies, materials and setup concepts. In addition, within the last two years, sustainability and End-of-Life (EoL) management started to gain major importance within PV technologies. In that sense, the Austrian flagship project "Sustainabe Photovoltaics - PVRe" aims to increase the sustainability of electricity generation from PV. One of the major issues are defect solder joints (socalled "cold solder joints"). This failure is mostly resulting from solder joint cracks, which in turn can be accountable for burn marks, hotspots, arcing discharges and of course substantial power losses.

This paper deals with the analysis method of the solder joint quality control. Since the cells are fully encapsulated, a conducted measurement method for analyzing reasons is impossible. So, one particularly well-suited noninvasive method is eddy current testing, which is mainly used for detecting cracks in surfaces, but can also be used for electrical conductivity measurements, [1], [2]. The method is based on the physical phenomenon of electromagnetic induction. If a wire coil that is supplied by an alternating current thus generating an oscillating magnetic primary field is brought closely to a conductive material, a circular flow of electrons, which is known as eddy current, will appear in the conductor, refer to Fig. 1. The eddy current will in turn generate its own secondary magnetic field, which interacts with the wire coil described through mutual inductance, [3].

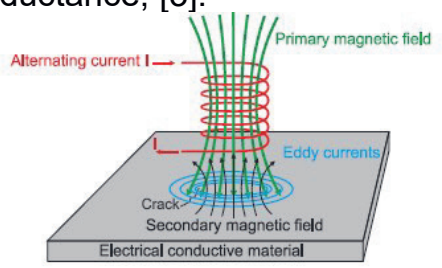

Fig. 1. Primary and secondary magnetic field generated by eddy currents in an electrically conductive material, [1].

Any change in the thickness of the metal, or other disturbances like changes in the conductivity alter the amplitude and pattern of the eddy current and its resulting magnetic field. This leads to a varying impedance of the coil, that is measured to determine the nature of the disturbance, [4]. This kind of non-destructive testing strongly depends on the penetration depth, which is influenced by the frequency of the measurement system as well as the magnetic permeability and conductivity of the test material. While the eddy current density is highest near the surface, the probing depth can be steered through frequency variation. Probing depths of several millimeters can be achieved in nonmagnetic materials, which makes this method an ideal candidate for our purposes as wires, con- 
nectors and solder joints in PV cells have typical thicknesses in the order of max. $0.3 \mathrm{~mm}$. Eddy current measurements are always calibrated according to an appropriate reference standard like air, or to a known good sample.

\section{Measurement Setup}

As probe a handmade air coil was developed with a diameter of $12 \mathrm{~mm}$ and a length of $10 \mathrm{~mm}$, which resulted in an inductivity value of $76 \mu \mathrm{H}$, refer to Fig. 2 a.). This sensing element was connected to an impedance analyzer E4990A from Keysight, which was set to the desired frequency range of $1 \mathrm{kHz}$ to $5 \mathrm{kHz}$. Eddy current measurement systems are influenced by several other effects in addition to the material properties: Most important are the distance to the device under test (DUT), which is referenced to the dimensions of the probe, as well as the alignment to it, but also vibrations or movements of the sensor probe during the measurement have to be avoided. When applying this technology to PV modules, the setup is well defined, because the probe can be pressed onto the backsheet of the PV module during the measurement, so the distance remains constant and even the position is set, leading to stable and repeatable results.

\section{Measurement Results}

To verify the system setup, a single cell was laminated identically as it is used in a PV module, see Fig. 2 b.). Two interconnections (busbar ribbon \& cross-connector) on the upper side were not soldered (red), while the other ones were soldered properly (green).

a.)
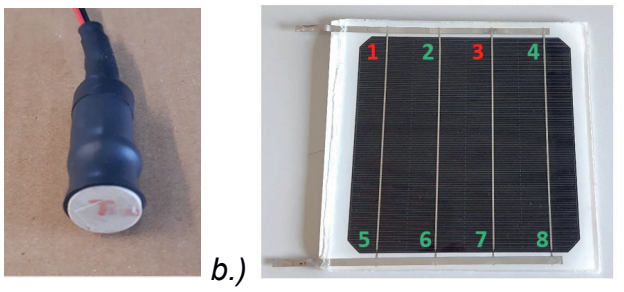

Fig. 2. a.) Sensor probe, b.) Test-cell with soldered and not soldered junctions for system verification.

To simulate a degradation of the cell due to aging effects it was artificially aged running through 100 thermal cycles from $-40^{\circ} \mathrm{C}$ to $+80^{\circ} \mathrm{C}$, according to the international standard IEC 61215. This degradation effect was analyzed by electroluminescence method and quantified by greyscale values, shown in Fig. 3.

For each measurement, the probe was located over a solder joint one after the other. Additionally, a measurement without a target was performed that depicted the reference for normalizing the result. In a post processing step, the variation between the $1 \mathrm{kHz}$ and $5 \mathrm{kHz}$ result was calculated, according to the theoretical equations. Finally, the results were correlated to the electroluminescence image of the cell. The bright shining regions illustrate a higher current flow due to better contacts between the wires, while the darker regions lead to lower currents due to higher resistances or lower conductivities. Tab. 1 presents the achieved results of all solder joints after processing. Due to the fact of small variations, a region of fluctuation is noticed, while the bad junctions show a significant increase in value.

\section{Table 1. Analyzed junction conductivity}

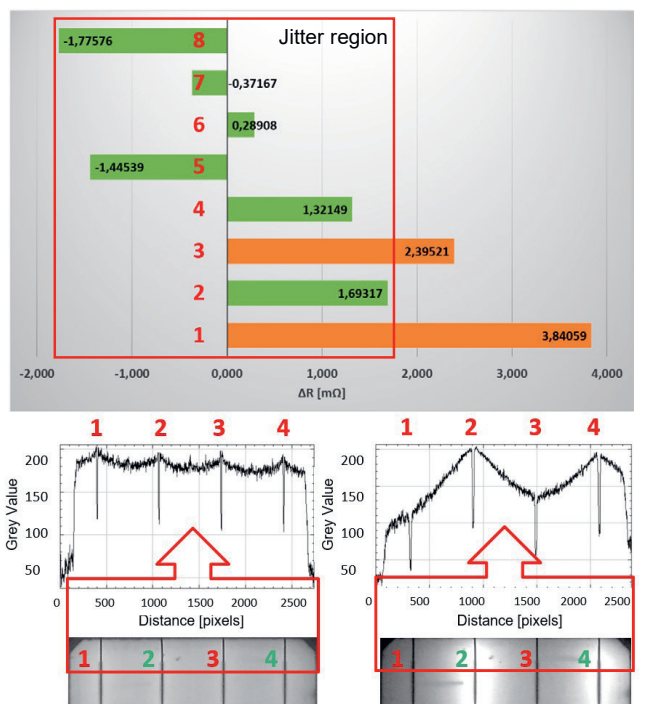

Fig. 3. Electroluminescence measurements of the PV cell before (left) and after thermal aging (right).

\section{Conclusion}

The presented multi frequency eddy current measurement method and achieved postprocessing calculation results are in good agreement with the electroluminescence investigations of a PV cell.

\section{Acknowledgement}

This work was conducted as part of the Austrian "Energy Research Program" project PVRe2 Sustainable Photovoltaics, funded by the Austrian Climate and Energy Fund and the Austrian Research Promotion Agency (FFG), both are gratefully acknowledged.

\section{References}

[1] L. Janousek, K. Capova, N. Yusa and K. Miya, "Multiprobe inspection for enhancing sizing ability in eddy current nondestructive testing", IEEE Trans. Magn. 2008, vol. 44, pp. 1618-1621.

[2] M. K. Markelov, A. S. Ishkov and A. V. Svetlov, "Eddy Current Measuring System for Testing Conductive Objects", 2020, pp. 1-5, doi: 10.1109/MWENT47943.2020.9067468.

[3] R. Pohl, A. Erhard, H. J. Montag, H. M. Thomas and $H$. Wüstenberg, "NDT techniques for railroad wheel and gauge corner inspection", NDT E Int. 2004, pp. 89-94.

[4] D. Placko and I. Dufour, "Eddy current sensors for nondestructive inspection of graphite composite materials", In Proceedings of the IEEE Conference of the Industry Applications Society (IAS'92), Houston, TX, USA, October 1992; pp. 1676-16 